\title{
A virtual trainer concept for robot-assisted human motor learning in rowing
}

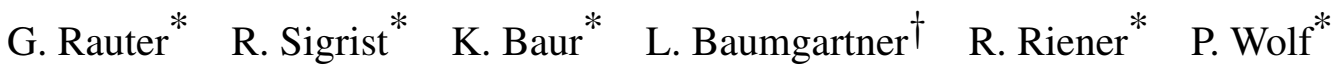 \\ (*) SMS Lab, Institute of Robotics and Intelligent Systems (IRIS), ETH Zurich, \\ and Medical Faculty, University of Zurich, Switzerland \\ (†) Hocoma AG, Volketswil, Switzerland \\ E-mail:rauter@mavt.ethz.ch,roland.sigrist@mavt.ethz.ch,kilianbaur@gmx.net, \\ lukas.baumgartner@hocoma.com,riener@mavt.ethz.ch,wolf@mavt.ethz.ch
}

\begin{abstract}
Keeping the attention level and observing multiple physiological and biomechanical variables at the same time at high precision is very challenging for human trainers. Concurrent augmented feedback, which is suggested to enhance motor learning in complex motor tasks, can also hardly be provided by a human trainer.

Thus, in this paper, a concept for a virtual trainer is presented that may overcome the limits of a human trainer. The intended virtual trainer will be implemented in a CAVE providing auditory, visual and haptic cues. As a first application, the virtual trainer will be used in a realistic scenario for sweep rowing. To provide individual feedback to each rower, the virtual trainer quantifies errors and provides concurrent auditory, visual, and haptic feedback. The concurrent feedback will be adapted according to the actual performance, individual maximal rowing velocity, and the athlete's individual perception.
\end{abstract}

\section{Introduction}

The goal of every sports trainer is to provide the optimal training for each athlete. Accordingly, the trainer has to know the perfect movement, the challenges which come along with complex movements, and the differences between skill levels. However, a human trainer is limited. Firstly, a human cannot hold the same level of attention and concentration over a longer period of time. Secondly, a trainer is not able to observe all important physiological and biomechanical variables characterizing the movement, neither at the same time nor at high precision. Thirdly, a trainer can hardly provide augmented concurrent feedback (=extrinsic feedback during the movement in real-time) which has been found to be beneficial to enhance motor learning in complex motor tasks [13,8].

To overcome the limits of a human trainer, we introduce a virtual trainer concept for robot-assisted human motor learning: the $\mathrm{M}^{3}$-trainer. The $\mathrm{M}^{3}$-trainer is intended to provide concurrent feedback in three modalities and to adapt to the individual perception of the human athlete. We hypothesize that this adaption of the $\mathrm{M}^{3}$-trainer to the individual athlete is a key feature to optimize human motor learning. Our hypothesis is also supported by the daily work of e.g. physiotherapists in hospitals and clinics: They adapt to individual needs, constraints, and are able to challenge subjects within their abilities. Thus, also the $\mathrm{M}^{3}$-trainer will select feedback in a way that the currently most important movement errors are addressed. Furthermore, also the feedback modality will be adapted in order to maximize the overall performance under consideration of the individual maximal capability of the athlete. As a first application, the $\mathrm{M}^{3}$-trainer will be tested on a high fidelity rowing simulator in a CAVE (Cave Augmented Virtual Environment) [10, 7, 11, 9, 6, 12, 5] (Figure 1).

In this paper, we present the basic principles applied by the $\mathrm{M}^{3}$-trainer to classify human movement errors in order to be able to select adequate feedback strategies for human motor learning. Furthermore, an insight into our idea to select appropriate concurrent feedback modalities and strategies are given. Concerning the haptic feedback strategies, we focus on usercooperative haptic feedback applied by a tendon-based parallel robot connected to the rowing simulator's outer oar end $[10,9,6,5]$.

This is an Open Access article distributed under the terms of the Creative Commons Attribution-Noncommercial License 3.0, which permits unrestricted use, distribution, and reproduction in any noncommercial medium, provided the original work is properly cited. 


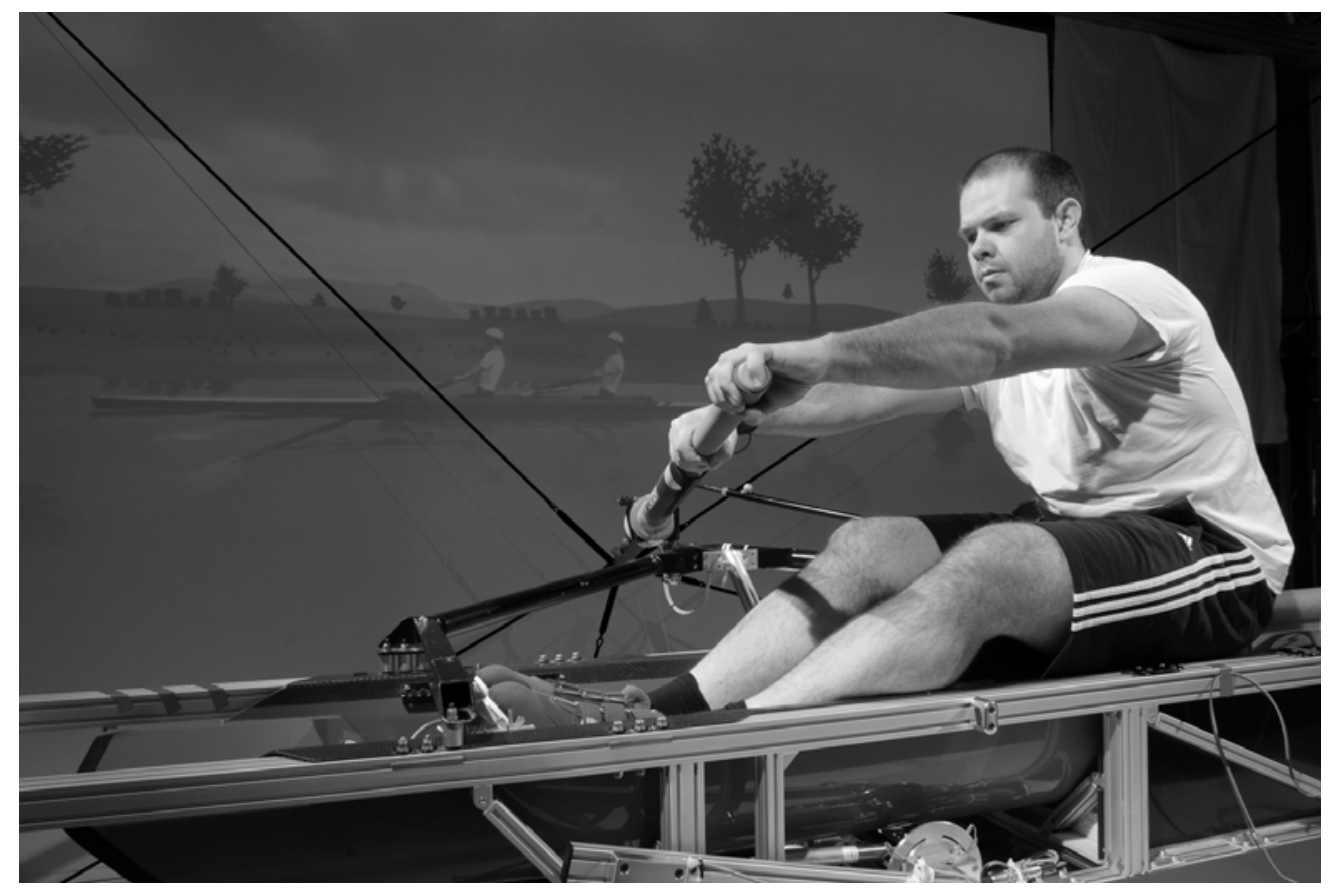

Figure 1: The rowing simulator is placed within an interactive VR-scenario providing auditory, visual and haptic cues.

\section{Automated Human Motor Learning with the $\mathbf{M}^{3}$-trainer}

During conventional training, an athlete performs a task and obtains feedback by a trainer after task completion. Due to the feedback on the errors of the last task execution, the athlete can constantly improve. Similar to this cycle of task execution and feedback, the control scheme of the $\mathrm{M}^{3}$-trainer was developed for a sweep rowing task (Figure 2).

During the training session, the rower is seated inside a shortened rowing skiff and holds a shortened oar. The skiff is placed in the middle of a CAVE providing a realistic interactive VR-scenario for sweep rowing $[10,7,11,12]$. Visual cues are provided via projections onto three screens surrounding the user $(4 \mathrm{~m} \times 3 \mathrm{~m}$, projectors: Projection Design F3+, Norway). Auditory cues are rendered using a ring of loudspeakers for wave field synthesis (112 speakers and 4 subwoofers, Iosono $\mathrm{GmbH}$, Erfurt, Germany). Haptic cues are rendered by a versatile tendon-based parallel robot (SMS Lab, IRIS, ETH Zurich) that is connected to the outer end of the shortened oar. In this way, the rower sees, hears, and feels the interaction with the virtual rowing scenario.

To enhance motor learning in this interactive VRscenario, the $\mathrm{M}^{3}$-trainer has been developed. This $\mathrm{M}^{3}$ trainer uses the sensors of the different components installed in the CAVE i.e.: a tendon-based parallel robot, an instrumented foot stretcher in the rowing boat [1], and an optelectrical motion tracking system (10 cameras, Qualisys, Gothenburg, Sweden). In order to classify rowing errors, the $\mathrm{M}^{3}$-trainer compares like a human one the actually observed movement of an athlete with "perfect" reference data. In our sweep rowing project, the reference data for error classification was obtained from measurements on professional rowers. The error classification of the actual rowing cycle will be done in real-time by an algorithm for online data analysis. This algorithm normalizes the measured data of a rowing cycle to reduce influences of speed and the athlete's anatomy. In a further step, all variables of a rowing cycle are transformed into Fourier space by FFT (Fast Fourier Transform) and reduced to a relevant amount of information applying PCA (Principal Component Analysis). The classification of rowing errors is then obtained using SVMs (Support Vector Machines). Consequently, the $\mathrm{M}^{3}$-trainer can choose the feedback modality and strategy based on the rowing error that was recognized (Modality \& Strategy Selection). In this way, the $\mathrm{M}^{3}$-trainer can provide visual, auditory and haptic feedback to the athlete about the actually recognized error. During training, the subject's errors together with the feedback given are stored in a subject's profile. This subject profile in combination with the athlete's individual skills and perceptual abilities will be the basis to modify the feedback modality 


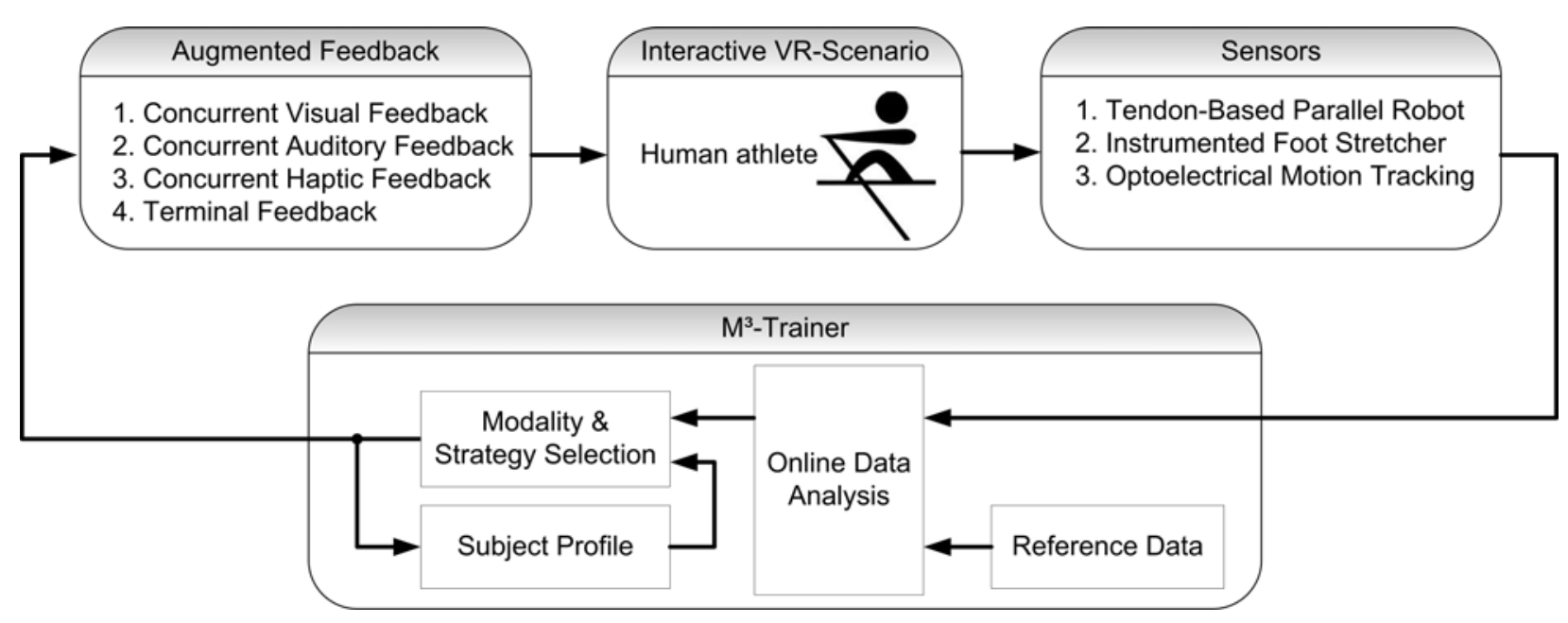

Figure 2: Training in an interactive VR-scenario enhanced by the $\mathrm{M}^{3}$-trainer

and strategy. In particular, the modification is based on the user's learning gradient as a reaction to the feedback and based on the individual potential for skill improvement through comparison of the theoretical individual optimal performance to the actual performance [3].

In contrast to first concepts of automated human motor learning in the field of behavioral research e.g. [2], the $\mathrm{M}^{3}$-trainer will not only be able to apply visual and auditory feedback [4], but also haptic feedback [6, 5] on the actual motor performance. Furthermore, the feedback modality and strategy will automatically be adapted to the individual performance and perception of each athlete.

\section{References}

[1] D. Krumm, M. Simnacher, G. Rauter, A. Brunschweiler, S. Odenwald, R. Riener, and P. Wolf. High-fidelity device for online recording of footstretcher forces during rowing. Procedia Engineering, 2:2721-2726, 2010.

[2] M. Lopez-Garate, A. Lozano-Rodero, and L. Matey. An adaptive and customizable feedback system for vr-based training simulators. In Proceedings of the 7th international joint conference on Autonomous agents and multiagent systems-Volume 3, pages 1635-1638. International Foundation for Autonomous Agents and Multiagent Systems Richland, SC, 2008.

[3] G. Rauter, L. Baumgartner, J. Denoth, R. Riener, and P. Wolf. Optimisation of the mean boat velocity in rowing. Computer methods in biomechanics and biomedical engineering, page 1, 2011.
[4] G. Rauter, A. Brunschweiler, M. Wellner, J. von Zitzewitz, R. Riener, and P. Wolf. Rowing novices can only partly profit from acoustic and visual display of a reference movement of an oar blade. In Progress in Motor Control VII, Marseille, France, 2009.

[5] G. Rauter, R. Sigrist, L. Marchal-Crespo, H. Vallery, R. Riener, and P. Wolf. Assistance or challenge? filling a gap in user-cooperative control. In Intelligent Robots and Systems (IROS), 2011 IEEE/RSJ International Conference on. IEEE.

[6] G. Rauter, J. von Zitzewitz, A. Duschau-Wicke, H. Vallery, and R. Riener. A tendon based parallel robot applied to motor learning in sports. In Proceedings of the IEEE BioRob, Tokyo, Japan, 2010.

[7] R. Sigrist. Influence of virtual audience on psychophysiological processes in rowing. Master's thesis, ETH Zurich, 2008.

[8] E. Todorov, R. Shadmehr, and E. Bizzi. Augmented feedback presented in a virtual environment accelerates learning of a difficult motor task. J Mot Behav, 29(2):147-158, 1997.

[9] J. von Zitzewitz, G. Rauter, R. Steiner, A. Brunschweiler, and R. Riener. A versatile wire robot concept as a haptic interface for sport simulation. In Proceedings of the 2009 IEEE International Conference on Robotics and Automation (ICRA), pages 313-318, 2009.

[10] J. von Zitzewitz, P. Wolf, V. Novakovic, M. Wellner, G. Rauter, A. Brunschweiler, and R. Riener. 
Real-time rowing simulator with multimodal feedback. Sports Technol, 1(6):257-266, 2008.

[11] M. Wellner, A. Schaufelberger, J. Zitzewitz, and R. Riener. Evaluation of visual and auditory feedback in virtual obstacle walking. Presence, 17(5):512-524, 2008.

[12] M. Wellner, R. Sigrist, and R. Riener. Virtual competitors influence rowers. PRESENCE: Teleoperators and Virtual Environments, 19(4):313-330, 2010.

[13] G. Wulf and C. H. Shea. Principles derived from the study of simple skills do not generalize to complex skill learning. Psychon Bull Rev, 9(2):185$211,2002$. 